\title{
Addition of apatinib to transarterial chemoembolization leads to improved outcome in patients with intermediate and advanced hepatocellular cancer
}

\author{
Pauline Viviane Pocha ${ }^{1}$, Chencheng Xie ${ }^{2,3}$, Christine Pocha ${ }^{2,3}$ \\ ${ }^{1}$ School of Medicine, University College of Dublin, Dublin, Ireland; ${ }^{2}$ Department of Medicine, Avera McKennan Hospital and University Center, \\ Sioux Falls, SD, USA; ${ }^{3}$ Sanford School of Medicine, Sanford School of Medicine, University of South Dakota, Vermillion, SD, USA \\ Correspondence to: Christine Pocha, MD, PhD, MPH. Avera Transplant Institute, Plaza 3, Suite 1200, 1315 S. Cliff Avenue, Sioux Falls, SD 57105, \\ USA. Email: christine.pocha@avera.org; christine.pocha@usd.edu. \\ Comment on: Zhao S, Zhang T, Dou W, et al. A comparison of transcatheter arterial chemoembolization used with and without apatinib for \\ intermediate- to advanced-stage hepatocellular carcinoma: a systematic review and meta-analysis. Ann Transl Med 2020;8:542.
}

Submitted Nov 18, 2020. Accepted for publication Dec 02, 2020.

doi: $10.21037 /$ atm-20-7534

View this article at: http://dx.doi.org/10.21037/atm-20-7534

The article by Zhao and colleagues is the first published systematic review and metanalysis of the combined therapy of transarterial chemoembolization (TACE) and apatinib for intermediate and advanced hepatocellular cancer (HCC).

Primary liver cancer with HCC accounting for $90 \%$ of all cases represents the most common cancer in male and female. Globally, approximately 854,000 new cases were reported. Overall, HCC has a poor prognosis and high mortality with 810,000 deaths per year (1).

Detecting tumors at an early and potential curative stage would be ideal, however screening measures are not widely and consistently implemented. Biomarkers for early detection are lacking. Outcome of surveillance programs are affected by lead-time bias hampering true effect on mortality and cost-effectivity, although surveillance remains strongly recommended. Prognosis of HCC patients depend on several factors: (I) tumor stage accounting for size and number of lesions, presence of vascular invasions and/or extrahepatic metastasis; (II) liver function [Child-TurcottePugh's score (CTP) based on bilirubin, albumin, portal hypertension, ascites]; (III) overall health (performance status, presence of symptoms). In clinical practice, patients are often identified at intermediate and advanced tumor stage limiting the use of curative measures.

The Barcelona Clinic Liver Cancer (BCLC) staging system which is widely accepted across the globe approves TACE as preferred treatment for HCC at the intermediate stage (B) (2). These patients have multinodular disease without vascular invasion, preserved liver function (CTP A without ascites), and are asymptomatic with a good performance status. TACE has been shown to prolong survival of these patients to up to 2.5 years. Applying the principle of stage mitigation, TACE can be applied to treat more advanced tumors (BCLC C) in highly selected patients as well as in patients with earlier tumors unsuitable to other therapies based on multidisciplinary tumor board decisions. TACE has been successfully used to downstage tumors outside accepted criteria for liver transplantation in patients who have proven to be otherwise suitable candidates for transplantation (3).

Despite being broadly used, TACE lacks standardization and an agreement on best techniques has not been reached. Delivery systems, frequency of applications as well as preferred chemotherapy drugs vary widely. TACE combines intra-arterial injection of a chemotherapeutic drug directly into the tumor and embolization of the feeding artery resulting in cytotoxicity and ischemia. The actual therapeutic effects may depend on the chemotherapeutic agents being applied. Drug-loaded microspheres promise a more tumor-targeted drug delivery and a sustained embolization effect (4). A recent metanalysis comparing various chemotherapeutic regimens combined with TACE for treatment of HCC concluded that the combination of an anthracycline, mitomycin, and pyrimidine provided the best results with regards to therapeutic response, adverse events and overall survival (OS) rate (probability: $\mathrm{P}=0.45$, 


\section{$0.32,0.35$, respectively) (5).}

Overall, unsatisfactory long-term treatment outcome with high rates of tumor recurrence is fueling the continuous search for better treatment modalities for intermediate and advanced HCC. Systemic therapy for patients with advanced HCC was introduced in 2007 with the survival benefit of sorafenib demonstrated in the SHARP and Asia Pacific trials $(6,7)$. Further clinical trials investigating the use of lenvatinib and regorafenib as well as ramucirumab and cabozantinib were successfully completed in 2017 and 2018, respectively (8). Multiple phase 3 randomized controlled trials of immune checkpoint inhibitors and combination therapies of those with molecular targeted agents are currently being conducted with the hope to change the treatment paradigm of HCC in the near future.

It is well documented that TACE induced hypoxemia increases expression of vascular endothelial growth factor (VEGF) in the remaining HCC tissue leading to accelerated neo-angiogenesis which may be responsible for treatment failure and/or relapse (9). Angiogenesis increases supply of oxygen and nutrients to HCC cells; a known critical factor involved in HCC development, growth and metastasis. The addition of tyrosine kinase inhibitors to TACE as adjuvant to suppress vessel growth seems logical.

Sorafenib was the first multikinase inhibitor approved for use in HCC. Sorafenib affects multiple molecular processes including cell signaling, angiogenesis, cell proliferation, and apoptosis. It inhibits a multitude of kinases including receptor tyrosine kinases, platelet-derived kinases and the Raf serine-threonine kinase. Secondary, it blocks growth factors such as c-KIT, RET, FLT-3, and receptor-beta (PDGFR- $\beta$ ) as well as VEGF receptor 1-3 (10). To date reported combination therapies of TACE plus sorafenib have provided mixed results. Some studies in patients with unresectable HCC provided encouraging data on survival benefits and safety of this combination $(11,12)$. Ren et al. reported improved median OS in the TACE plus sorafenib receiving group compared to the TACE alone group $(29.0 \pm 7.2$ versus $14.9 \pm 1.1$ months; $\mathrm{P}=0.008)$ (13). Contradictory, another phase 3 study as well as a metaanalysis showed no improvement in OS $(14,15)$. Most recently, the TACTICS trial which included 33 institutions in Japan reported a median progression free survival (PFS) and until time to untreatable (unTACEable) progression (TTUP) which was significantly better in the TACE plus sorafenib group than in the TACE alone group (25.2 versus 13.5 months; $\mathrm{P}=0.006)$ and (26.7 versus 20.6 months;
$\mathrm{P}=0.02$ ), respectively. The reported 1- and 2-year survival were $96.2 \%$ and $82.7 \%$ and $77.2 \%$ and $64.6 \%$, respectively in the TACE plus sorafenib group compared to the TACE alone group (16).

To date, the last word has not been spoken, yet if ultimately the combination of TACE and sorafenib in patients with unresectable HCC will lead to improved survival. Increased level of VEGF expression in HCC cells is related to resistance to sorafenib and overall poor prognosis. The unmet need to explore additional targeted therapies for intermediate and advanced HCC remains.

Regorafenib was approved by the FDA in 2017 as second line drug for the treatment of HCC in patients who failed sorafenib therapy. RESORCE was a worldwide randomized controlled trial including 152 sites in 21 countries investigating regorafenib in patients with HCC and preserved liver function (CTP A) who progressed on sorafenib. The investigators reported a significant overall survival benefit (hazard ratio: 0.63; 95\% CI, 0.50-0.79; one-sided $\mathrm{P}<0.0001)$. The reported median survival was 10.6 months (95\% CI, 9.1-12.1 months) for patients receiving regorafenib compared to 7.8 (6.3-8.8) months for patients receiving placebo. Regorafenib had an acceptable tolerability profile $(17,18)$.

Apatinib is a vascular endothelial growth factor receptor 2 tyrosine kinase inhibitor (VEGFR2-TKI) with promising therapeutic potential in various cancers including gastric, non-small cell lung and HCC (19). Preclinical studies on a human HCC xenograft mice model (20) confirmed a direct suppressive effect of apatinib on HCC by several pathways inhibiting tumor cell viability, proliferation, migration, as well as increased apoptosis $(\mathrm{P}<0.05)$. An impressive $71 \%$ inhibition ratio $(\mathrm{P}<0.05)$ was reported. The underlying mechanisms were thought to be inhibition of AKT phosphorylation and significantly downregulated phosphorylation levels of several tyrosine kinase receptors (IGF-R, PDGFR- $\alpha$ ). Li et al. summarized that apatinib may hold promise for patients with HCC because of its multitarget tyrosine kinase receptor inhibition implying a direct anti-HCC effect (20).

In this manuscript, Han et al. are starting off by highlighting a case of a male patient from China chronically infected with hepatitis B who suffered a spontaneous rupture of an HCC. The patient needed emergency surgery resulting in a partial hepatectomy. Subsequently, he was treated with TACE plus sorafenib but developed resistance and his tumor progressed. The addition of apatinib resulted in 19 months of added life (21). 
Yang et al. (22) compared 25 patients with progressive HCC on TACE who were able to receive apatinib offlabel with 22 patients treated with TACE alone. Addition of apatinib led to significantly improved OS and PFS for BCLC B and C patients. Fatigue, hypertension, hand-foot syndrome, weight loss, and anorexia were more commonly seen in the TACE plus apatinib group. Overall, adverse events were felt to be manageable and tolerable. The documented survival benefit was not associated with age, gender, or ECOC performance status. As expected, patients with macro-vascular tumor invasion and/or extrahepatic metastasis had worse outcome.

Given these promising preliminary data on apatinib, Zhao and colleagues conducted a comprehensive retrospective evaluation of the impact of the addition of apatinib to TACE in patients with intermediate and advanced stage HCC. After rigorous selection of studies their final analysis included 1,342 patients from 23 studies. Inclusion criteria were defined as (I) intermediate or advanced stage HCC by imaging (CT, MRI) or pathology, (II) treatment with TACE plus apatinib or TACE alone, and (III) clearly reported outcome measures using mRECIST criteria. Analyzed outcome measures included complete response (CR), partial response (PR), stable disease (SD) as well as progressive disease (PD). Survival rates at half year and one year were used when available. Zhao et al. found that adverse events were reported to be tolerable; but common and multifaceted in the TACE plus apatinib patients. Most commonly reported were fever, abdominal pain, nausea, vomiting, myelosuppression, hypertension, hand-foot-syndrome, proteinuria, diarrhea, and oral ulcer. Dosing of apatinib ranged from $250 \mathrm{mg}$ daily, $500 \mathrm{mg}$ daily to $850 \mathrm{mg}$ daily. The drug was combined with various TACE regimens including 5 -fluouracil plus adriamycin (4 studies), adriamycin (2 studies), adriamycin plus platinol (7 studies), and 5 -fluouracil plus adriamycin + platinol (4 studies). The authors found that survival rates at half year (OR 2.741, 95\% CI, 1.745-4.306) and at one year (OR $2.284,95 \%$ CI, 2.099-3.429) were significantly better in patients receiving TACE plus apatinib compared to TACE alone. The TACE plus apatinib combination appeared to have an improved objective response rate (OR 2.683, 95\% CI, 2.099-3.429), lower disease progression rate (OR 0.341, 95\% CI, 0.255-0.456) as well as an overall better disease control rate (OR 2.919, 95\% CI, 2.194-3.903). Therefore, the authors speculate that apatinib may be a viable treatment option for intermediate and advanced HCC patients who express sorafenib resistance. Apatinib may be particularly useful in those tumors expressing high levels of vascular endothelial growth factors.

In their discussion, the authors acknowledged some of the shortcoming of (I) the current treatment paradigm for intermediate and advanced HCC and (II) of their own analysis.

(I) It has been widely accepted that TACE has some beneficial short-term efficacy but long-term efficacy remains unsatisfactory. Improving outcome and survival for those HCC patients is urgently needed. It is of utmost importance to clearly define and identify the patients in whom treatment will improve survival but also keeping treatment related risks and adverse events aligned. According to the BCLC, TACE is recommend as preferred therapy for intermediate stage HCC (2). In patients with advanced stage HCC which is complicated by vascular invasion and/or extrahepatic metastasis, the preference is given to systemic therapy. It is also well accepted that the ability of the BCLC to clearly stratify all HCC patients for clinical trials as well as for daily clinical practice appears somewhat limited. Researcher and clinicians alike are urging further stratifications within each class. Proposed further subclassification may include a more detailed assessment of the patient's liver function (CTP score, presence of ascites, incorporation of additional scoring systems such as ALBI), molecular and other prognostic biomarkers as well as other patient' specific features such as development and degree of systemic therapy related side effects and pattern of progression of disease $(2,12,23)$.

(II) The authors admit to methodological limitations of their own analysis which may affect the statistical power of the analysis. Some studies which were included provided incomplete data on safety and efficacy including lack of reporting of VEGF or AFP. Reported follow up overall was short. In addition, included studies exclusively came from the Eastern part of the world potentially resulting in a regional bias as population and tumor related factors such as etiology of HCC, prevalence of cirrhosis, comorbidities, and overall treatment approach differ in Eastern and Western parts of the world. Publication bias which is applicable to all systematic reviews or literature analyses has to be considered as well as often negative studies are being withheld from publications by the investigators. 
In summary, Zhao et al. provided the first and comprehensive review concluding that that a combination therapy of TACE plus apatinib could be considered as a viable treatment option for patients with intermediate and advanced HCC. Further data and ideally randomized controlled trials are needed to identify the most suitable patient population with regards to tumor stage (intermediate, advanced) and other characteristics (molecular markers including VEGF), most effective TACE technique and chemotherapy cocktail, as well as optimal dose of apatinib to improve outcome parameters and survival for these HCC patients while minimizing adverse events.

\section{Acknowledgments}

Funding: None.

\section{Footnote}

Provenance and Peer Review: This article was commissioned by the editorial office, Annals of Translational Medicine. The article did not undergo external peer review.

Conflicts of Interest: The authors have completed the ICMJE uniform disclosure form (available at http://dx.doi. org/10.21037/atm-20-7534). The authors have no conflicts of interests to declare.

Ethical Statement: The authors are accountable for all aspects of the work in ensuring that questions related to the accuracy or integrity of any part of the work are appropriately investigated and resolved.

Open Access Statement: This is an Open Access article distributed in accordance with the Creative Commons Attribution-NonCommercial-NoDerivs 4.0 International License (CC BY-NC-ND 4.0), which permits the noncommercial replication and distribution of the article with the strict proviso that no changes or edits are made and the original work is properly cited (including links to both the formal publication through the relevant DOI and the license). See: https://creativecommons.org/licenses/by-nc-nd/4.0/.

\section{References}

1. Akinyemiju T, Abera S, Ahmed M, et al. The Burden of Primary Liver Cancer and Underlying Etiologies From
1990 to 2015 at the Global, Regional, and National Level: Results From the Global Burden of Disease Study 2015. JAMA Oncol 2017;3:1683-91.

2. EASL Clinical Practice Guidelines: Management of hepatocellular carcinoma. J Hepatol 2018;69:182-236.

3. Parikh ND, Waljee AK, Singal AG. Downstaging hepatocellular carcinoma: A systematic review and pooled analysis. Liver Transpl 2015;21:1142-52.

4. Melchiorre F, Patella F, Pescatori L, et al. DEB-TACE: a standard review. Future Oncol 2018;14:2969-84.

5. Guo T, Wu P, Liu P, et al. Identifying the Best Anticancer Agent Combination in TACE for HCC Patients: A Network Meta-analysis. J Cancer 2018;9:2640-9.

6. Llovet JM, Ricci S, Mazzaferro V, et al. Sorafenib in advanced hepatocellular carcinoma. $\mathrm{N}$ Engl J Med 2008;359:378-90.

7. Cheng AL, Kang YK, Chen Z, et al. Efficacy and safety of sorafenib in patients in the Asia-Pacific region with advanced hepatocellular carcinoma: a phase III randomised, double-blind, placebo-controlled trial. Lancet Oncol 2009;10:25-34.

8. Llovet JM, Montal R, Sia D, et al. Molecular therapies and precision medicine for hepatocellular carcinoma. Nat Rev Clin Oncol 2018;15:599-616.

9. Sacco R, Tapete G, Simonetti N, et al. Transarterial chemoembolization for the treatment of hepatocellular carcinoma: a review. J Hepatocell Carcinoma 2017;4:105-10.

10. Gauthier A, Ho M. Role of sorafenib in the treatment of advanced hepatocellular carcinoma: An update. Hepatol Res 2013;43:147-54.

11. Cabrera R, Pannu DS, Caridi J, et al. The combination of sorafenib with transarterial chemoembolisation for hepatocellular carcinoma. Aliment Pharmacol Ther 2011;34:205-13.

12. Geschwind JF, Kudo M, Marrero JA, et al. TACE Treatment in Patients with Sorafenib-treated Unresectable Hepatocellular Carcinoma in Clinical Practice: Final Analysis of GIDEON. Radiology 2016;279:630-40.

13. Ren B, Wang W, Shen J, et al. Transarterial Chemoembolization (TACE) Combined with Sorafenib versus TACE Alone for Unresectable Hepatocellular Carcinoma: A Propensity Score Matching Study. J Cancer 2019;10:1189-96.

14. Meyer T, Fox R, Ma YT, et al. Sorafenib in combination with transarterial chemoembolisation in patients with unresectable hepatocellular carcinoma (TACE 2): a randomised placebo-controlled, double-blind, phase 3 
trial. Lancet Gastroenterol Hepatol 2017;2:565-75.

15. Li L, Zhao W, Wang M, et al. Transarterial chemoembolization plus sorafenib for the management of unresectable hepatocellular carcinoma: a systematic review and meta-analysis. BMC Gastroenterol 2018;18:138.

16. Kudo M, Ueshima K, Ikeda M, et al. Randomised, multicentre prospective trial of transarterial chemoembolisation (TACE) plus sorafenib as compared with TACE alone in patients with hepatocellular carcinoma: TACTICS trial. Gut 2020;69:1492-501.

17. Ettrich TJ, Seufferlein T. Regorafenib. Recent Results Cancer Res 2018;211:45-56.

18. Bruix J, Qin S, Merle P, et al. Regorafenib for patients with hepatocellular carcinoma who progressed on sorafenib treatment (RESORCE): a randomised, double-blind, placebo-controlled, phase 3 trial. Lancet 2017;389:56-66.

19. Xue JM, Astère M, Zhong MX, et al. Efficacy and safety of apatinib treatment for gastric cancer, hepatocellular

Cite this article as: Pocha PV, Xie C, Pocha C. Addition of apatinib to transarterial chemoembolization leads to improved outcome in patients with intermediate and advanced hepatocellular cancer. Ann Transl Med 2021;9(1):2. doi: 10.21037/atm-20-7534 carcinoma and non-small cell lung cancer: a meta-analysis. Onco Targets Ther 2018;11:6119-28.

20. Li X, Xu A, Li H, et al. Novel role of apatinib as a multi-target RTK inhibitor in the direct suppression of hepatocellular carcinoma cells. Biochim Biophys Acta Mol Basis Dis 2018;1864:1693-701.

21. Han Z, He Z, Wang C, et al. The effect of apatinib in the treatment of sorafenib resistant metastatic hepatocellular carcinoma: A case report. Medicine (Baltimore) 2018;97:e13388.

22. Yang Z, Chen G, Cui Y, et al. The safety and efficacy of TACE combined with apatinib on patients with advanced hepatocellular carcinoma: a retrospective study. Cancer Biol Ther 2019;20:321-7.

23. Kudo M, Arizumi T, Ueshima K. Subclassification of BCLC B Stage Hepatocellular Carcinoma and Treatment Strategies: Proposal of Modified Bolondi's Subclassification (Kinki Criteria). Dig Dis 2015;33:751-8. 